\title{
Successful Synthesis of Fully Aromatic Polyketones via Nickel Complex-Mediated Aromatic Coupling Polymerization of Bis(chlorobenzoylated) $o$-Terphenyls
}

\author{
Katsuya Maeyama, Tadahiro OHe, Hiroyuki NaKamura, and \\ Noriyuki YONEZAWA ${ }^{\dagger}$ \\ Department of Organic and Polymer Materials Chemistry, Tokyo University of Agriculture \\ and Technology, Koganei, Tokyo 184-8588, Japan
}

(Received October 17, 2002; Accepted January 10, 2003)

\begin{abstract}
KEY WORDS Fully Aromatic Polyketone / Wholly Aromatic Polyketone / o-Terphenylene Unit / Aromatic Coupling Polymerization / Nickel Complex-Mediated Reaction /
\end{abstract}

Wholly aromatic polyketone is a type of aromatic polyketone characterized by the absence of a hetero linkage in the main chain. The polymer skeleton composed of only arylene rings and ketonic carbonyl groups has still considerable room for a variety of further molecular designing and modifications. Though there have been reported numerous papers concerning the synthesis of aromatic polyketones, ${ }^{1-7}$ almost all of them have been classified as aromatic poly(ether ketone)s. To the best of our knowledge, there have been reported only four papers ${ }^{8-11}$ concerning the synthesis of wholly aromatic polyketones besides our work. ${ }^{12-16}$

The small number of examples of wholly aromatic polyketones is plausibly due to the following two synthetic obstacles. One is the inherent limitation of bondforming methods and the other is the absence of flexibilization assistance by ether linkage. From the point of bond formation methods, nucleophilic aromatic substitution of the phenoxide ion is the most efficient synthetic method for aromatic poly(ether ketone)s. It is, however, inapplicable to the synthesis of wholly aromatic polyketones. In this respect, synthetic approaches for wholly aromatic polyketones are practically restricted to either of the following two carboncarbon bond formation modes: bond formation between an aromatic carbon and a carboxy one and that between aromatic carbons. As it is difficult to introduce two acyl groups on an aromatic system via electrophilic aromatic acylation, this protocol requires severe reaction conditions resulting in poor regioselective introduction of the aroyl groups. From the point of structural characteristics of the polymer skeleton, the lack of ether linkage plausibly regidifies the polymer main chain thus inducing aggregation of the intermediate oligomers, resulting in depression of the polymerization degree.

To overcome these difficulties, appropriate monomer designing to permit sustainable reactivity and regioselectivity is needed. One solution we have achieved is the use of 2,2'-dimethoxybiphenyl as an acyl-acceptant monomer, which successfully gave a high-molecularweight, wholly aromatic polyketone through electrophilic acylation polymerization. ${ }^{12}$ Presumably, 2,2'dimethoxybiphenyl promotes smooth and highly regioselective progress of electrophilic aromatic acylation polymerization and, at the same time, the twisted biphenylene of 2,2'-dimethoxy-5,5'-biphenylene repeating unit suppresses aggregation of the resulting polyketones. ${ }^{12}$ This structural advantage has enabled the synthesis of wholly aromatic polyketones via nickel complex-mediated aromatic coupling polymerization of $5,5^{\prime}$-bis(chlorobenzoylated) $2,2^{\prime}-$ dimethoxybiphenyls. ${ }^{13,14}$

As the next stage in the course of this synthetic study, we planned to synthesize 'fully' aromatic polyketones where ether bonds are excluded perfectly from the molecule. In this communication, we wish to report the successful synthesis of 'fully' aromatic polyketones and their unexpectedly high thermal resistance.

Two isomeric, fully aromatic polyketones composed of only phenylene units and ketonic carbonyl groups $(\mathbf{2 a}, \mathbf{b})$ were obtained through nickel complexmediated aromatic coupling polymerization ${ }^{17-20}$ of $\operatorname{bis}(p$-chlorobenzoylated) $o$-terphenyl (1a) or $\operatorname{bis}(m$ chlorobenzoylated) $o$-terphenyl (1b), prepared by the $\mathrm{P}_{2} \mathrm{O}_{5}-\mathrm{MsOH}$ mediated regioselective diaroylation of $o$ -

${ }^{\dagger}$ To whom correspondence should be addressed (E-mail: yonezawa@cc.tuat.ac.jp). 

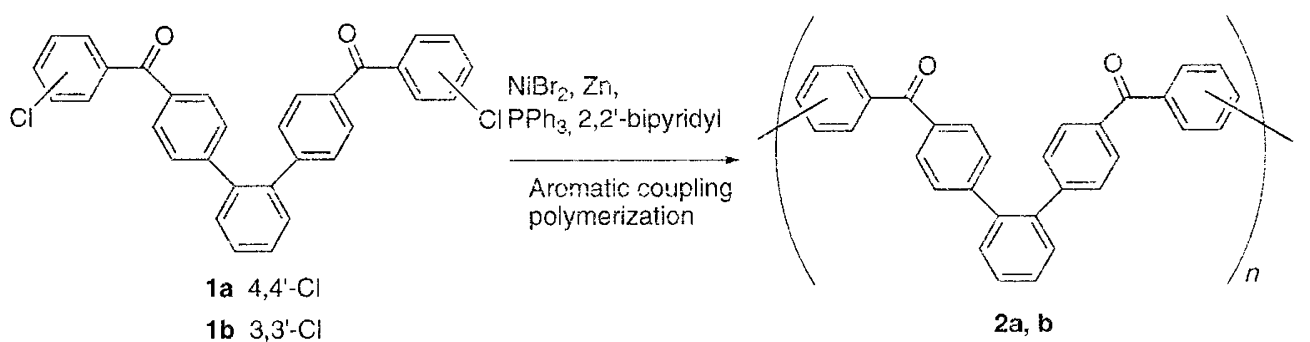

Scheme 1. Synthesis of fully aromatic polyketones containing $o$-terphenylene unit.

terphenyl. ${ }^{21}$

Nickel complex-mediated aromatic coupling reaction of monomer 1a in an aprotic polar solvent such as DMAc or DMF proceeded smoothly to give the fully aromatic polyketone (2a). The optimum result was obtained when monomer 1a was allowed to react in the presence of half an equimolar amount of $\mathrm{NiBr}_{2}, 3.1$ molar amounts of $\mathrm{Zn}$, half an equimolar amount of 2,2' bipyridyl (bpy), and an equimolar amount of $\mathrm{PPh}_{3}$ in DMAc at $100^{\circ} \mathrm{C}$ for $2 \mathrm{~h}$. On the contrary, when $\mathrm{CHCl}_{3}$ or 1,4-dioxane was employed as a solvent, monomer 1a was quantitatively recovered. The contrastive behavior between the reactions in these solvents is probably caused by the difference in solubility of the nickel intermediates to each solvent.

Polyketone 2a obtained from the reaction under the optimized conditions has molecular weight $\left(M_{\mathrm{n}}\right)^{22}$ of 8000 and inherent viscosity $\left(\eta_{\text {inh }}\right)^{23}$ of $0.23 \mathrm{dL} \mathrm{g}^{-1}$. It gave a transparent and colorless flexible film by casting from chloroform. The resulting polymer (2a) shows higher solubility in organic solvents than the wholly aromatic polyketones containing the $2,2^{\prime}$-dimethoxy$5,5^{\prime}$-biphenylene unit which we have previously reported. ${ }^{12-14}$ Actually, the resulting polymer (2a) is soluble not only in halogenated solvents such as chloroform and chlorobenzene but also in ethereal solvents such as 1,4-dioxane and THF. Furthermore, it is soluble in toluene, DMF, and DMSO by heating.

The optimum conditions for polymerization of the $m$-chlorobenzoylated $o$-terphenyl monomer (1b) are essentially the same as those for monomer 1a, and the obtained polyketone (2b) has $M_{\mathrm{n}}$ of 8000 and $\eta_{\text {inh }}$ of $0.19 \mathrm{dL} \mathrm{g}^{-1}$. However, the resulting polymer (2b) has lower solubility in organic solvents such as chloroform than polymer $\mathbf{2 a}$.

Both polymers $\mathbf{2 a}$ and $\mathbf{2 b}$ have amorphous nature. The glass transition temperatures, 176 and $156^{\circ} \mathrm{C}$, respectively, are a little lower than those of the previously reported wholly aromatic polyketones containing the 2,2'-dimethoxy-5,5'-biphenylene unit (Figure 1)..$^{14}$ The relatively lower $T_{\mathrm{g}}$ 's of polyketones $\mathbf{2 a}$ and $\mathbf{2 b}$ compared to the case of the 2,2'-dimethoxy-5, $5^{\prime}$ biphenylene units in polyketones $\mathbf{3} \mathbf{a}$ and $\mathbf{3 b}$ are presum-
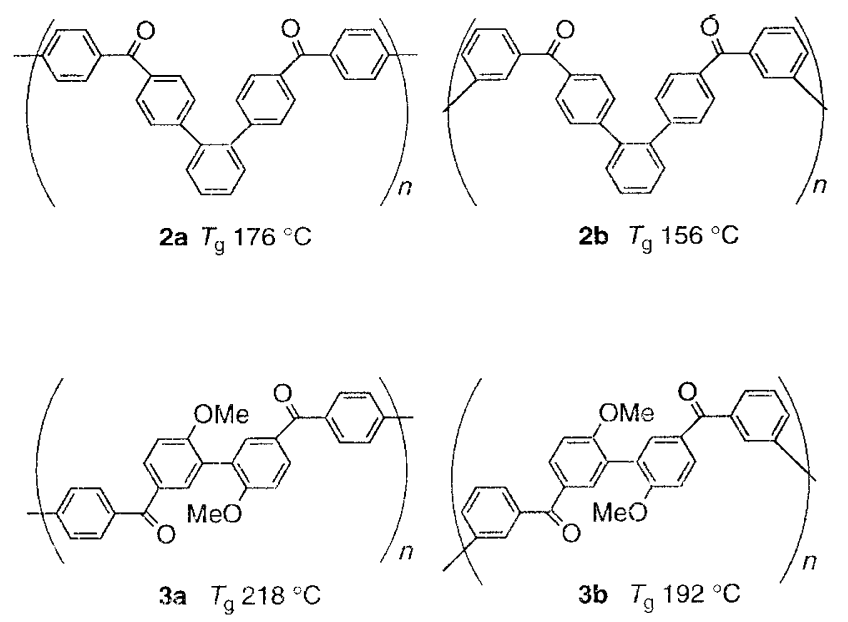

Figure 1. Wholly aromatic polyketones and their $T_{\mathrm{g}}$ 's.

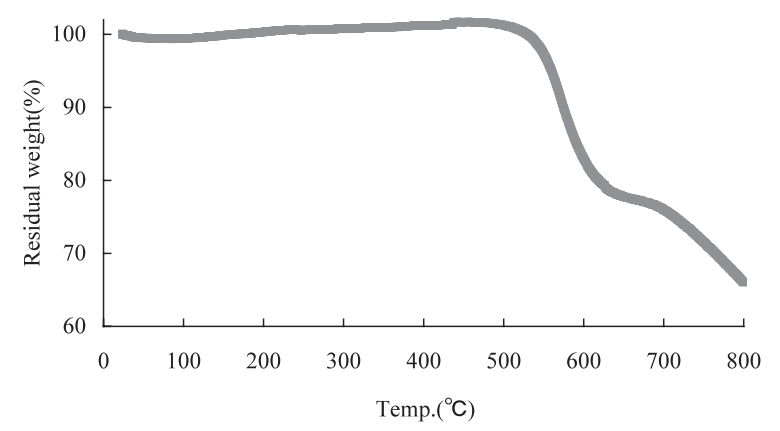

Figure 2. TGA thermogram of polyketone 2a.

ably due to their larger free volume brought about by the specific three-dimensional structure around the $o$ terphenylene units. In addition, the polyketones having the $p, p^{\prime}$-biphenylenedicarbonyl unit (2a and $\mathbf{3 a}$ ) are shown to have higher $T_{\mathrm{g}}$ than the corresponding $m, m^{\prime}$ biphenylenedicarbonyl isomeric polyketone ( $2 \mathbf{b}$ and $\mathbf{3 b}$ ), which is presumably due to the more symmetrical, rod-like, and rigid structure of the $p, p^{\prime}$-biphenylenecontaining unit compared to the $m, m^{\prime}$-biphenylene one.

Thermogravimetric analysis of polyketones $\mathbf{2 a}$ and 2b disclosed excellent thermal stability of these polyketones (Figure 2). No reduction of weight in the temperature range up to $c a .500^{\circ} \mathrm{C}$ and reduction of $10 \mathrm{wt} \%$ of the weight at $c a .550^{\circ} \mathrm{C}$ are observed. The thermal stability of these polyketones is far beyond our expectation based on the thermal behavior of polyketones 3a and $\mathbf{3 b}$ where thermal decomposition was observed at 
ca. $450{ }^{\circ} \mathrm{C} .{ }^{24}$

The successful synthesis of fully aromatic polyketones 2, their remarkable thermal resistance, high $T_{\mathrm{g}}$, and specific solution behavior are interpreted by the structural assistance of the $o$-terphenylene unit.

The twisted crankshaft-shaped steric structure of the $o$-terphenylene unit should inhibit the stiff aggregation of the polymer chain, thus enabling the bond elongation reaction to proceed satisfactorily. The high solubility of these polyketones to various organic solvents is also considered to depend on the disordered structure induced by the $o$-terphenylene unit in the same manner. Similar solubility behavior is observed for poly(ether ketone)s and polyamides containing the $o$-terphenylene unit prepared by Akutsu and coworkers. ${ }^{25,26}$ As mentioned above, it is also considered that the inhibition of stiff aggregation of the polymer chain by twisting of alignment of the phenylene rings in the $o$-terphenylene unit facilitates the solvation and, moreover, increases the flexibility of polymer chains to depress the $T_{\mathrm{g}}$ to some degree.

On the other hand, the high thermal resistance is interpreted to be caused by other factors. It is thought that exclusion of the carbon-hetero atom bond in the whole molecule repels the disconnection points, then affords the cumulative stability of the polyketone molecules.

Acknowledgment. This work was supported by SANEYOSHI SCHOLARSHIP FOUNDATION and the Ministry of Education, Culture, Sports, Science, and Technology of Japan.

\section{REFERENCES}

1. T. E. Attwood, P. C. Dawson, J. L. Freeman, L. R. J. Hoy, J. B. Rose, and P. A. Staniland, Polymer, 22, 1096 (1981).

2. M. J. Mullins and E. P. Woo, J. Macromol. Sci. Rev. Macromol. Chem. Phys., 27, 313 (1987).

3. P. A. Staniland, in "Comprehensive Polymer Science", 2nd ed, G. C. Eastmond, A. Ledwith, S. Russo, and P. Sigwalt, Ed., Pergamon Press, Oxford, 1989, vol. 5, p 483.

4. A. Pandya, J. Yang, and H. W. Gibson, Macromolecules, 27, 1367 (1994).

5. N. Yonezawa, J. Synth. Org. Chem. Jpn., 53, 172 (1995).

6. V. Lakshmana Rao, J. Macromol. Sci. Rev. Macromol. Chem. Phys., 35, 661 (1995).

7. N. Yonezawa, S. Mori, S. Miyata, Y. Ueha-Anyashiki, and K. Maeyama, React. Funct. Polym., 53, 11 (2002).

8. M. Bochmann and K. Kelley, J. Chem. Soc., Chem. Commun., 532 (1989).

9. M. Rehahn, A.-D. Schlueter, and G. Wegner, Makromol.
Chem., Rapid. Commun., 11, 535 (1990).

10. J. S. Moore, Makromol. Chem., Rapid. Commun., 13, 91 (1992).

11. M. Bochmann and J. Lu, J. Polym. Sci., Part A: Polym. Chem., 32, 2493 (1994).

12. N. Yonezawa, S. Miyata, T. Nakamura, S. Mori, Y. Ueha, and R. Katakai, Macromolecules, 26, 5262 (1993).

13. N. Yonezawa, T. Namie, T. Ikezaki, T. Hino, H. Nakamura, Y. Tokita, and R. Katakai, React. Funct. Polym., 30, 261 (1996).

14. N. Yonezawa, T. Ikezaki, H. Nakamura, and K. Maeyama, Macromolecules, 33, 8125 (2000).

15. K. Maeyama, T. Namie, H. Nakamura, and N. Yonezawa, Recent Progress in Polycondensation, 173 (2002).

16. N. Yonezawa, H. Nakamura, and K. Maeyama, React. Funct. Polym., 51, 19 (2002).

17. M. Ueda and F. Ichikawa, Macromolecules, 23, 926 (1990).

18. M. Ueda, Y. Seino, and J. Sugiyama, Polym. J., 25, 1319 (1993).

19. M. Ueda, Y. Seino, Y. Haneda, M. Yoneda, and J. Sugiyama, J. Polym. Sci., Part A: Polym. Chem., 32, 675 (1994).

20. Representative polymerization procedure is as follows: In a $30 \mathrm{~mL}$ two-necked round flask, monomer $(2,63.0 \mathrm{mg}$, $0.125 \mathrm{mmol})$, nickel bromide (II) $(13.7 \mathrm{mg}, 0.0625 \mathrm{mmol}$ ), triphenylphosphine (32.8 mg, $0.125 \mathrm{mmol}), 2,2^{\prime}$-bipyridyl ( $9.7 \mathrm{mg}, 0.0625 \mathrm{mmol})$, and zinc $(25.2 \mathrm{mg}, 0.388 \mathrm{mmol})$ were placed and freshly distilled DMAc was added via a syringe. The reaction mixture was stirred using a stirring bar in DMAc at $100^{\circ} \mathrm{C}$ for $2 \mathrm{~h}$. The mixture was diluted with DMAc and poured into a $1 / 9(\mathrm{v} / \mathrm{v})$ hydrochloric acid/methanol solution with vigorous stirring to separate out a solid. The solid was separated from the solution and washed with methanol and acetone in turn. The insoluble fraction was dried under reduced pressure at $80^{\circ} \mathrm{C}$ giving polymer 2. Polymer 2a: IR (KBr): 1657, 1603, 1399, 1312, 1277, 928, $754 \mathrm{~cm}^{-1}$. ${ }^{1} \mathrm{H}$ NMR $\delta\left(\mathrm{CDCl}_{3}\right): 7.30-7.34(4 \mathrm{H}, \mathrm{m}), 7.52(4 \mathrm{H}$, pseudo s), 7.70-7.75(8 H, m), 7.88-7.90(4 H, m)ppm. ${ }^{13} \mathrm{C}$ NMR $\delta$ $\left(\mathrm{CDCl}_{3}\right): 127.2,127.3,128.3,128.4,129.7,129.8,129.9$, 129.9, 130.0, 130.7, 130.8, 132.4, 135.7, 137.0, 139.5, 143.7, 145.6, 195.6 ppm. Calcd. For $\left(\mathrm{C}_{32} \mathrm{H}_{20} \mathrm{O}_{2}\right)_{\mathrm{n}}, \mathrm{C} 88.04, \mathrm{H} 4.62 \%$. Found. C 87.05, H 4.98\%.

21. N. Yonezawa, H. Nakamura, T. Ohe, and K. Maeyama, Synth. Commun., in press.

22. Number average molecular weight $\left(M_{\mathrm{n}}\right)$ of the polyketone was estimated by polystyrene calibration using GPC measurement.

23. The inherent viscosity $\left(\eta_{\text {inh }}\right)$ of the polyketone was measured at a concentration of $0.3 \mathrm{~g} \mathrm{dL}^{-1}$ in conc. $\mathrm{H}_{2} \mathrm{SO}_{4}$ at $30^{\circ} \mathrm{C}$ using an Ostwald viscometer.

24. N. Yonezawa, S. Miyata, S. Mori, Y. Ueha-Anyashiki, K. Aizawa, and K. Maeyama, submitted.

25. F. Akutsu, K. Takahashi, Y. Kasashima, M. Inoki, and K. Naruchi, Macromol. Rapid. Commun., 16, 495 (1995).

26. F. Akutsu, M. Inoki, K. Takahashi, T. Yonemura, Y. Kasashima, and K. Naruchi, Polym. J., 28, 1107 (1996). 